\title{
A Pilot Included Column Mean Vanishing Matrix
}

\author{
Myungsup $\mathrm{Kim}^{1}$, Do Y. Kwak ${ }^{2}$ \\ 1 Tunitel, Kwan Pyong Dong, Yusung-Ku, Daejeon, Korea \\ ${ }^{2}$ Department of Mathematical Sciences, Korea Advanced Institute of Science and Technology, Daejeon, Korea \\ Correspondence: Do Y. Kwak, Department of Mathematical Sciences, Korea Advanced Institute of Science and Technol- \\ ogy, Daejeon, Korea, 34141. E-mail: kdy@kaist.ac.kr.
}

Received: November 16, 2016 Accepted: March 8, 2017 Online Published: March 23, 2017

doi:10.5539/jmr.v9n2p128 URL: https://doi.org/10.5539/jmr.v9n2p128

\begin{abstract}
In this paper, we study a special matrix used in OFDM technology including the pilot vector. This is based on the property of 'column mean vanishing' and orthogonal columns. We study the spectral decomposition. Using this, we suggest a new method of generating such matrices. Numerical examples are included.
\end{abstract}

Keywords: pilot, orthogonal columns, column mean vanishing property, SVD

\section{Introduction}

Recently, there have arisen a large necessity of developing a new technology in wireless communications. An OFDM or its generalization is a big trend (O. Edfors, et al., 1998; Frederiksen, F. B. \& Prasad, R., 2002; Myungsup, K. \& Kwak, D. Y.). In this paper, we review the algorithm developed in (Myungsup, K. \& Kwak, D. Y.) and study some properties of the OFDM matrix. Based on this we propose a simple method to generate the matrix. In the resulting matrix, we see the pilot column has only two nonzero entries which correspond to zero rows, so that the pilot does not interfere with other data.

Definition 1.1. We say a matrix A has a column mean vanishing (CMV) property if the sum of each column is zero.

\section{Generation of CMV Matrix Having Orthonormal Columns}

Let $L=n \gg N$. Recall the scheme introduced in (Myungsup, K. \& Kwak, D. Y.):

\section{Algorithm Orth-pilot}

1. Given a $N \times(N-2)$ initial matrix $K_{p}$ with orthonormal columns.

2. Multiply by $L \times N$ matrix $\mathbf{P}$ obtaining $A=\mathbf{P} K_{p}$.

3. Perform IFFT to obtain $\mathbf{F}^{-1}\left(\mathbf{P} K_{p}\right)$.

4. Subtract the first row from all the rows, the result is $\mathbf{\Phi} \circ \mathbf{F}^{-1}\left(\mathbf{P} K_{p}\right)$.

5. Perform FFT to get $\mathbf{F} \circ \mathbf{\Phi} \circ \mathbf{F}^{-1}\left(\mathbf{P} K_{p}\right)$.

6. Multiply $\mathbf{P}^{T}$ to obtain $\hat{K}:=\mathbf{P}^{T} \circ \mathbf{F} \circ \boldsymbol{\Phi} \circ \mathbf{F}^{-1}\left(\mathbf{P} K_{p}\right)$.

7. Let $G=U V^{H}$ where $U \Sigma V^{H}$ is the SVD of $\hat{K}$.

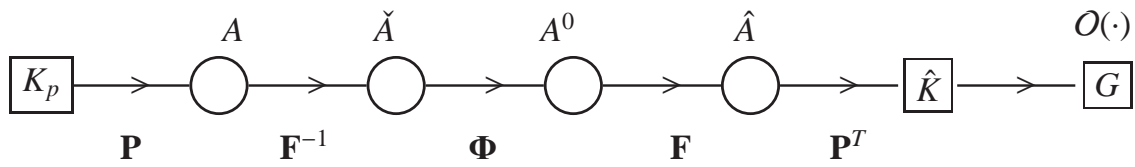

Figure 1. Signal flow diagram for matrix generation. $O(\cdot)$ is orthonormalization operator.

Now we will explain more details of the algorithm: 


\section{Step (2). Permute and Pad Zeros}

Assume $N=2 m+1$ is odd. Set $M=N-2, L=n \gg N$. Starting from an $N \times M$ initial matrix, we construct an $L \times M$ matrix as follows: Move the last $m+1$ rows of $K_{p}$ to the first $m+1$ rows of $K_{p}$. Next fill it with pad with $L-M$ zero rows (called zero padding). This process can be expressed as $\mathbf{P} K_{p}$ where

$$
\mathbf{P}=\left[\begin{array}{c|c}
\mathbf{0}_{(m+1) \times m} & \mathbf{I}_{m+1} \\
\hline 0 & 0 \\
\vdots & \vdots \\
0 & 0 \\
\hline \mathbf{I}_{m} & \mathbf{0}_{m \times(m+1)}
\end{array}\right]
$$

Steps (3) and (4) : IFFT Followed by Subtraction of the First Row

Let us use the notation $K=\left(k_{i j}\right)$ and $K_{1}=\left(k_{i j}^{1}\right):=\mathbf{P} K_{p}$. Let $\check{K}_{1}=\mathbf{F}^{-1}\left(\mathbf{P} K_{p}\right)$ be the inverse FFT of $\mathbf{P} K_{p}$. By definition of IFFT the first row of $\check{K}_{1}$ is

$$
\check{\mathbf{k}}_{1}=\left[\breve{k}_{11}, \check{k}_{12}, \cdots, \breve{k}_{1 M}\right]=\frac{1}{n}\left[\sum_{i=0}^{n-1} k_{i 1}^{1}, \sum_{i=0}^{n-1} k_{i 2}^{1}, \cdots, \sum_{i=0}^{n-1} k_{i M}^{1}\right] .
$$

Hence the matrix after step (4) is

$$
\check{K}_{1}{ }^{\prime}=\left[\begin{array}{cccc}
\check{k}_{11} & \check{k}_{12} & \ldots & \check{k}_{1 M} \\
\check{k}_{21} & \check{k}_{22} & \ldots & \check{k}_{2 M} \\
\vdots & \vdots & \ldots & \vdots \\
\check{k}_{n, 1} & \check{k}_{n, 2} & \cdots & \check{k}_{n, M}
\end{array}\right]-\left[\begin{array}{cccc}
\check{k}_{11} & \check{k}_{12} & \ldots & \check{k}_{1 M} \\
\check{k}_{11} & \check{k}_{12} & \ldots & \check{k}_{1 M} \\
\vdots & \vdots & \ldots & \vdots \\
\check{k}_{11} & \check{k}_{12} & \cdots & \check{k}_{1 M}
\end{array}\right] \equiv \check{K}_{1}-\check{K}_{1}^{*}
$$

Here $\check{K}_{1}^{*}$ is the matrix all of whose rows are the vector $\check{\mathbf{k}}_{1}$.

Lemma 2.1. The sum of all entries of each column of the matrix $\hat{K}$ is zero.

Proof. Clear from (2) and (3).

Step (7) - Nearest Orthogonal Matrix

The scheme to find the nearest orthogonal matrix (Higham, N. J. 1986; R. -C. Li., 1995; Ji-Guang, S., 1995; Banerjee, 2014) is given by

$$
G=U V^{H}=\hat{K}\left(\hat{K}^{H} \hat{K}\right)^{-1 / 2}
$$

where $U \Sigma V^{H}$ is the SVD of $\hat{K}$.

Theorem 2.1. The matrix G obtained in step (7) satisfies CMV property:

Proof. Let $\overrightarrow{\mathbf{1}}=[1, \cdots, 1]$. Then by Lemma 2.1 , we have

$$
\overrightarrow{\mathbf{1}} \cdot \hat{K}=[0,0, \cdots, 0] .
$$

Hence by (4) we see

$$
\overrightarrow{\mathbf{1}} \cdot G=\overrightarrow{\mathbf{1}} \cdot \hat{K}\left(\hat{K}^{H} \hat{K}\right)^{-1 / 2}=[0,0, \cdots, 0]
$$




\section{Simplification of the Algorithm}

In this paper we simplify the algorithm above. We apply the algorithm to an initial matrix having CMV property. First consider the case $N=2 m+1$ is odd. We will explain with $m=2$, general case follows easily from this. Consider the following $N \times(N-1)$ initial matrix.

$$
K^{*}=\frac{1}{\sqrt{2}}\left[\begin{array}{cc|c|ccc}
0 & 0 & 0 & 0 & 1 & 1 \\
\hline 0 & 0 & 1 & 1 & 0 & 0 \\
1 & 1 & 0 & 0 & 0 & 0 \\
0 & 0 & 0 & 0 & 0 & 0 \\
1 & -1 & 0 & 0 & 0 & 0 \\
0 & 0 & 1 & -1 & 0 & 0 \\
\hline 0 & 0 & 0 & 0 & 1 & -1
\end{array}\right]
$$

Using this matrix we will generate a pilot included matrix having the desired properties. We remove a specified column (3rd, say) consisting of two 1's and move the next column to the first to get $N \times(N-2)$ matrix

$$
K_{t 1}=\frac{1}{\sqrt{2}}\left[\begin{array}{ccc|cc}
0 & 0 & 0 & 1 & 1 \\
\hline 0 & 0 & 1 & 0 & 0 \\
1 & 1 & 0 & 0 & 0 \\
0 & 0 & 0 & 0 & 0 \\
1 & -1 & 0 & 0 & 0 \\
0 & 0 & -1 & 0 & 0 \\
\hline 0 & 0 & 0 & 1 & -1
\end{array}\right] \Rightarrow K_{t 2}=\frac{1}{\sqrt{2}}\left[\begin{array}{c|cccc}
0 & 0 & 0 & 1 & 1 \\
\hline 1 & 0 & 0 & 0 & 0 \\
0 & 1 & 1 & 0 & 0 \\
0 & 0 & 0 & 0 & 0 \\
0 & 1 & -1 & 0 & 0 \\
-1 & 0 & 0 & 0 & 0 \\
\hline 0 & 0 & 0 & 1 & -1
\end{array}\right]
$$

Then subtract the row vector $\left[\begin{array}{lllll}0 & 2 & 0 & 2 & 0\end{array}\right]$ from the center row, to get

$$
K_{p, e}=\frac{1}{\sqrt{2}}\left[\begin{array}{c|cccc}
0 & 0 & 0 & 1 & 1 \\
\hline 1 & 0 & 0 & 0 & 0 \\
0 & 1 & 1 & 0 & 0 \\
0 & -2 & 0 & -2 & 0 \\
0 & 1 & -1 & 0 & 0 \\
-1 & 0 & 0 & 0 & 0 \\
\hline 0 & 0 & 0 & 1 & -1
\end{array}\right]
$$

In general it looks like this:

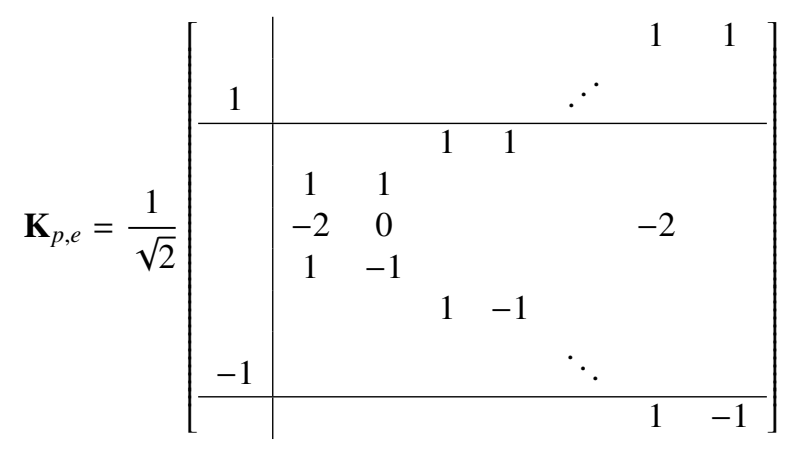

Next, we see the case $N=2 m$ (even). We start from $(N+1) \times N$ matrix. For example, when we want $8 \times 6$ matrix, we start from a $9 \times 8$ matrix

$$
\frac{1}{\sqrt{2}}\left[\begin{array}{cccc|c|ccc}
0 & 0 & 0 & 0 & 0 & 0 & 1 & 1 \\
0 & 0 & 0 & 0 & 1 & 1 & 0 & 0 \\
\hline 0 & 0 & 1 & 1 & 0 & 0 & 0 & 0 \\
1 & 1 & 0 & 0 & 0 & 0 & 0 & 0 \\
0 & 0 & 0 & 0 & 0 & 0 & 0 & 0 \\
1 & -1 & 0 & 0 & 0 & 0 & 0 & 0 \\
0 & 0 & 1 & -1 & 0 & 0 & 0 & 0 \\
\hline 0 & 0 & 0 & 0 & 1 & -1 & 0 & 0 \\
0 & 0 & 0 & 0 & 0 & 0 & 1 & -1
\end{array}\right]
$$


Remove the zero row in the center from (9) and remove first column. Then we remove a specified column (5-th, say) and move the next one to first to get $N \times(N-2)$ matrix

$$
K_{t 1}=\frac{1}{\sqrt{2}}\left[\begin{array}{ccc|c|ccc}
0 & 0 & 0 & 0 & 0 & 1 & 1 \\
0 & 0 & 0 & 1 & 1 & 0 & 0 \\
\hline 0 & 1 & 1 & 0 & 0 & 0 & 0 \\
1 & 0 & 0 & 0 & 0 & 0 & 0 \\
-1 & 0 & 0 & 0 & 0 & 0 & 0 \\
0 & 1 & -1 & 0 & 0 & 0 & 0 \\
\hline 0 & 0 & 0 & 1 & -1 & 0 & 0 \\
0 & 0 & 0 & 0 & 0 & 1 & -1
\end{array}\right] \Rightarrow K_{t 2}=\frac{1}{\sqrt{2}}\left[\begin{array}{c|ccccc}
0 & 0 & 0 & 0 & 1 & 1 \\
1 & 0 & 0 & 0 & 0 & 0 \\
\hline 0 & 0 & 1 & 1 & 0 & 0 \\
0 & 1 & 0 & 0 & 0 & 0 \\
0 & -1 & 0 & 0 & 0 & 0 \\
0 & 0 & 1 & -1 & 0 & 0 \\
\hline-1 & 0 & 0 & 0 & 0 & 0 \\
0 & 0 & 0 & 0 & 1 & -1
\end{array}\right]
$$

Then subtract the row vector $[0,0,1,0,1,0]$ from 4 -th and 5-th rows. The resulting matrix is the initial for pilot included matrix.

$$
K_{p, e}=\frac{1}{\sqrt{2}}\left[\begin{array}{c|ccccc}
0 & 0 & 0 & 0 & 1 & 1 \\
1 & 0 & 0 & 0 & 0 & 0 \\
\hline 0 & 0 & 1 & 1 & 0 & 0 \\
0 & 1 & -1 & 0 & -1 & 0 \\
0 & -1 & -1 & 0 & -1 & 0 \\
0 & 0 & 1 & -1 & 0 & 0 \\
\hline-1 & 0 & 0 & 0 & 0 & 0 \\
0 & 0 & 0 & 0 & 1 & -1
\end{array}\right]
$$

Lemma 3.1. If the initial matrix $K_{p}\left(K_{p, e}\right.$ or $\left.K_{p, o}\right)$ satisfies $C M V$ property, then steps (1)-(7) is simplified as

\section{Algorithm Orth-pilot-CMV}

1. Given a $N \times(N-2)$ initial $C M V$ matrix $K_{p}$ with orthonormal columns.

2. Let $G=U V^{H}$ where $U \Sigma V^{H}$ is the $S V D$ of $\hat{K}=K_{p}$.

\section{Property of Odd Columns}

We assume $N$ is odd. The case of even is similar. Let $\mathbf{k}_{i}$ and $\mathbf{g}_{i}$ and denote the $i$-th column of the matrix $K_{p}$ and $G$ respectively.

Lemma 4.1. The odd columns of $K_{p}$ are orthogonal to all other columns of $K_{p}$. As a consequence, for all odd $j$, the vector $\mathbf{e}_{j}=[0, \cdots, 1, \cdots, 0]^{T}$ is an eigenvector of $K_{p}^{H} K_{p}$ corresponding to the eigenvalue 1.

Proof. The orthogonality of odd columns of $K_{p}$ comes from that of $K^{*}$ of (5) since during the transformation of $K^{*}$ to $K_{p}$ in (5), (7), the odd columns did not change essentially(only the orders are permuted). Let $K_{p}=\left[\mathbf{k}_{1}, \cdots, \mathbf{k}_{N-2}\right]$. Then $K_{p} \mathbf{e}_{j}=\mathbf{k}_{j}$ and hence the $j$-th column of $\hat{K}_{p}^{H} \hat{K}_{p}$ satisfies

$$
\hat{K}_{p}^{H} \hat{K}_{p} \mathbf{e}_{j}=K_{p}^{H} K_{p} \mathbf{e}_{j}=\left[\begin{array}{c}
\mathbf{k}_{1}^{T} \\
\vdots \\
\mathbf{k}_{N-1}^{T}
\end{array}\right] \mathbf{k}_{j}=\left[\begin{array}{c}
\mathbf{k}_{1}^{T} \cdot \mathbf{k}_{j} \\
\vdots \\
\mathbf{k}_{N-1}^{T} \cdot \mathbf{k}_{j}
\end{array}\right]=\mathbf{e}_{j} .
$$

This means that when $j$ is odd, the $j$-th columns of $K_{p}$ are orthogonal to all other columns of $K_{p}$. Clearly (12) implies the second assertion of the lemma.

Example 4.1. For $N=5$ we see

$$
K_{p}^{H} K_{p}=\frac{1}{2}\left[\begin{array}{ccccc}
1 & 0 & 0 & 0 & -1 \\
0 & 1 & -2 & 1 & 0 \\
0 & 1 & 0 & -1 & 0
\end{array}\right]\left[\begin{array}{ccc}
1 & 0 & 0 \\
0 & 1 & 1 \\
0 & -2 & 0 \\
0 & 1 & -1 \\
-1 & 0 & 0
\end{array}\right]=\frac{1}{2}\left[\begin{array}{ccc}
2 & 0 & 0 \\
0 & 6 & 0 \\
0 & 0 & 2
\end{array}\right]
$$

Note that the zeros in the box keep the odd columns of $K_{p}$ orthogonal to other columns. In view of (12), $K_{p}^{H} K_{p}$ has two eigenvectors $\mathbf{e}_{j}, j=1,3$ corresponding to the eigenvalue 1 . 
Theorem 4.2. The odd columns of $G=K_{p}\left(K_{p}^{H} K_{p}\right)^{-1 / 2}$ are the same as those of $K_{p}$.

Proof. From the spectral decomposition of $K_{p}=U \Sigma V^{H}$ we have that of $K_{p}^{H} K_{p}$ :

$$
K_{p}^{H} K_{p}=V \Sigma^{H} \Sigma V^{H}:=V \Lambda V^{H}\left(V^{H}=V^{-1}\right),
$$

where by (12) $\Lambda$ and $V$ have the following form:

$$
\Lambda=\left[\begin{array}{ccccc}
1 & 0 & 0 & \cdots & 0 \\
0 & \lambda_{2} & 0 & \cdots & 0 \\
0 & 0 & 1 & & 0 \\
\cdots & & \cdots & \ddots & 0 \\
0 & 0 & 0 & \cdots & 1
\end{array}\right], \Lambda^{-1 / 2}=\left[\begin{array}{ccccc}
1 & 0 & 0 & \cdots & 0 \\
0 & \frac{1}{\lambda_{2}} & 0 & \cdots & 0 \\
0 & 0 & 1 & & 0 \\
\cdots & & \cdots & \ddots & 0 \\
0 & 0 & 0 & \cdots & 1
\end{array}\right], V=\left[\mathbf{e}_{1}, \mathbf{v}_{2}, \cdots, \mathbf{e}_{M-1}, \mathbf{v}_{M}\right]
$$

Note that for $j$ odd $V \mathbf{e}_{j}=\mathbf{e}_{j}$ and for each odd $j$,

$$
\begin{aligned}
K_{p}\left(K_{p}^{H} K_{p}\right)^{-1 / 2} \mathbf{e}_{j} & =K_{p} V \Lambda^{-1 / 2} V^{-1} \mathbf{e}_{j} \\
& =K_{p} V \Lambda^{-1 / 2} \mathbf{e}_{j} \\
& =K_{p} V \mathbf{e}_{j} \\
& =K_{p} \mathbf{e}_{j}
\end{aligned}
$$

This is the same as $j$-th column of $K_{p}$ (normalization does not change even columns).

\section{Numerical Example}

Example 5.1. Let $N=7$. We have, from initial matrix (7)

$$
G_{p}=\left[\begin{array}{ccccc}
0.0000 & -0.1954 & 0.0000 & 0.5117 & 0.7071 \\
0.7071 & 0.0000 & 0.0000 & 0.0000 & 0.0000 \\
0.0000 & 0.5117 & 0.7071 & -0.1954 & 0.0000 \\
0.0000 & -0.6325 & 0.0000 & -0.6325 & 0.0000 \\
0.0000 & 0.5117 & -0.7071 & -0.1954 & 0.0000 \\
-0.7071 & 0.0000 & 0.0000 & 0.0000 & 0.0000 \\
0.0000 & -0.1954 & 0.0000 & 0.5117 & -0.7071
\end{array}\right]
$$

Example 5.2 (Even $N)$. When $N=8, M=6$ we start from the initial matrix

$$
K_{p}=\frac{1}{\sqrt{2}}\left[\begin{array}{c|ccccc}
0 & 0 & 0 & 0 & 1 & 1 \\
1 & 0 & 0 & 0 & 0 & 0 \\
\hline 0 & 0 & 1 & 1 & 0 & 0 \\
0 & 1 & -1 & 0 & -1 & 0 \\
0 & -1 & -1 & 0 & -1 & 0 \\
0 & 0 & 1 & -1 & 0 & 0 \\
\hline-1 & 0 & 0 & 0 & 0 & 0 \\
0 & 0 & 0 & 0 & 1 & -1
\end{array}\right]
$$

to get

$$
G=\left[\begin{array}{cccccc}
0.0000 & 0.0000 & -0.1494 & 0.0000 & 0.5577 & 0.7071 \\
0.7071 & 0.0000 & 0.0000 & 0.0000 & 0.0000 & 0.0000 \\
0.0000 & 0.0000 & 0.5577 & 0.7071 & -0.1494 & 0.0000 \\
0.0000 & 0.7071 & -0.4082 & 0.0000 & -0.4082 & 0.0000 \\
0.0000 & -0.7071 & -0.4082 & 0.0000 & -0.4082 & 0.0000 \\
0.0000 & 0.0000 & 0.5577 & -0.7071 & -0.1494 & 0.0000 \\
-0.7071 & 0.0000 & 0.0000 & 0.0000 & 0.0000 & 0.0000 \\
0.0000 & 0.0000 & -0.1494 & 0.0000 & 0.5577 & -0.7071
\end{array}\right]
$$


In these matrices, we observe that rows corresponding to the nonzero entries of pilot vector (blue) are zeros (red) except the first entry.

\section{References}

Banerjee, Sudipto, \& Roy, Anindya, (2014). Linear Algebra and Matrix Analysis for Statistics, Texts in Statistical Science, Chapman and Hall/CRC.

Edfors, O., Sandell, M., van de Beek, J. -J., \& Wilson, S. K. (1998). OFDM channel estimation by singular value decomposition. IEEE Trans. Commun., 46, 931-939. https://doi.org/10.1109/26.701321

Fleming, B. F., \& Prasad, R. (2002). An Overview of OFDM and Related Techniques Towards Development of Future Wireless Multimedia Communications, in Radio and Wireless Conference, RAWCON 2002, 19-22.

Ji-Guang S. (1995). A Note on Backward Perturbations for the Hermitian Eigenvalue Problem, 385-393 of BIT 35.

Kim, M., \& Kwak, D. Y. Generalized OFDM, Submitted to IEEE Communications Letters.

Higham, N. J., (1986). Computing the Polar Decomposition - With Applications. SIAM J. SCI. STAT. COMPUT., 7(4). https://doi.org/10.1137/0907079

R.-C. Li., (1995). New Perturbation bounds for the polar factor. SIAM J. Matrix Anal. Appl.,16(1), 327-332. https://doi.org/10.1137/S0895479893256359

\section{Copyrights}

Copyright for this article is retained by the author(s), with first publication rights granted to the journal.

This is an open-access article distributed under the terms and conditions of the Creative Commons Attribution license (http://creativecommons.org/licenses/by/4.0/). 